\title{
Experimental Investigation on Reconstructing Sound Reflection Coefficient by NAH in Finite Space
}

\author{
Xiao Yan $^{1, a}$, Qiang Xuecai ${ }^{2, b}$, Shang Dejiang ${ }^{1, c}$, Liu Yongwei ${ }^{1, d}$ \\ ${ }^{1}$ Acoustic Science and Technology Laboratory, Harbin Engineering University, Harbin, China \\ 150001; College of Underwater Acoustic Engineering, Harbin Engineering University, Harbin, China \\ 150001 \\ ${ }^{2}$ CSIS N0.703 Research Institute, Harbin, China 150001 \\ axiaoyan_0220@126.com, bqiangxuecai4098@sina.com, c'shangdejiang@hrbeu.edu.cn, 'liuyongw \\ ei3000@hrbeu.edu.cn
}

Keywords: reflection coefficient; near-field acoustic holography (NAH); finite space; data preprocessing

\begin{abstract}
A pre-processing method for the holography measurement data is proposed to solve the problem caused by the multi-path interferences in finite space. In order to verify the effectiveness of this method, a standard steel plate is measured in an anechoic tank. The sound reflection coefficient of the steel plate is reconstructed by near-field acoustic holography (NAH) technology using the pre-processed data. The experimental results showed that: using the pre-processed data to reconstruct the sound reflection coefficient by NAH can effectively suppress the effect of multi-path interferences, and improve the measurement accuracy. And more, the requirements for the measurement environment are not very strict. Therefore, this pre-processing method will have broad application prospects.
\end{abstract}

\section{Introduction}

The sound reflection coefficient of material in a wide-angle range can be simultaneously obtained by near-field acoustic holography (NAH) technology [1, 2]. The NAH technology is widely used due to its convenience and quickness. Most researches on NAH measurement method are in the anechoic water tank, mainly aiming at free field or semi-space. If the tank is not completely anechoic, the effect of multi-path interferences on the NAH measurement results could not be ignored.

In view of this, the holography measurement data are pre-processed before holography reconstructing in this paper. The experimental researches are taken in an anechoic tank, the experimental results showed that: the data pre-processing could effectively reduce the measurement errors caused by multi-path interferences of the test field.

\section{The effect of multi-path interferences on holography reconstruction accuracy}

In the process of sound reflection coefficient reconstructing by NAH, the complex sound pressures on two holography planes between the source and the material are required. The complex sound pressures are decomposed into plane wave components by spatial Fourier transform. In a free space, the incident wave component and the reflected wave component on the material surface can be separated by plane wave propagation theory. And then, the sound reflection coefficient can be calculated as follows [1]:

$$
C_{r}\left(k_{x}, k_{y}\right)=\frac{P_{m}\left(k_{x}, k_{y}, z_{2}\right) \cdot \exp \left(j k_{z} z_{1}\right)-P_{m}\left(k_{x}, k_{y}, z_{1}\right) \cdot \exp \left(j k_{z} z_{2}\right)}{P_{m}\left(k_{x}, k_{y}, z_{1}\right) \cdot \exp \left(-j k_{z} z_{2}\right)-P_{m}\left(k_{x}, k_{y}, z_{2}\right) \cdot \exp \left(-j k_{z} z_{1}\right)}
$$

Where, $P_{m}\left(k_{x}, k_{y}, z_{1,2}\right)$ is the holography plane wave component, $z_{1,2}$ is the distance between holography plane and the material surface. 


$$
k_{z}=\sqrt{k^{2}-k_{x}^{2}-k_{y}^{2}} \quad(k=2 \pi f / c)
$$

The incidence angle $\theta$ is determined by $\left(k_{x}, k_{y}\right)$ :

$$
\theta=\arcsin \left(\sqrt{k_{x}^{2}+k_{y}^{2}} / k\right)
$$

It is supposed that there is only a point sound source but no material in the finite field, considering the effect of multi-path interferences on the measurement results, each holography plane wave component $P_{0}^{\prime}\left(k_{x}, k_{y}, z_{1,2}\right)$ can be expressed as follows:

$$
P_{0}^{\prime}\left(k_{x}, k_{y}, z_{1,2}\right)=P_{0}\left(k_{x}, k_{y}, z_{1,2}\right) * H_{0}\left(k_{x}, k_{y}, z_{1,2}\right)
$$

Where, "*" is the convolution symbol, $P_{0}\left(k_{x}, k_{y}, z_{1,2}\right)$ is the holography plane wave component in free space, $H_{0}\left(k_{x}, k_{y}, z_{1,2}\right)$ is the two-dimensional Fourier transform of the channel impulse response function in finite space.

After the material to be measured is placed in the finite field, the holography plane wave component $P_{m}^{\prime}\left(k_{x}, k_{y}, z_{1,2}\right)$ can be expressed:

$$
P_{m}^{\prime}\left(k_{x}, k_{y}, z_{1,2}\right)=P_{m}\left(k_{x}, k_{y}, z_{1,2}\right) * H_{m}\left(k_{x}, k_{y}, z_{1,2}\right)
$$

Where, $P_{m}\left(k_{x}, k_{y}, z_{1,2}\right)$ is the holography plane wave component when the material is placed in free space, $H_{m}\left(k_{x}, k_{y}, z_{1,2}\right)$ is the two-dimensional Fourier transform of the channel impulse response function when the material is placed in finite space.

Thus, the calculation formula of sound reflection coefficient is as follows [3]:

$$
C_{r}^{\prime}\left(k_{x}, k_{y}\right)=\frac{P_{2}^{\prime} \exp \left(j k_{z} z_{1}\right)-P_{1}^{\prime} \exp \left(j k_{z} z_{2}\right)}{P_{1}^{\prime} \exp \left(-j k_{z} z_{2}\right)-P_{2}^{\prime} \exp \left(-j k_{z} z_{1}\right)}=\frac{P_{2} * H_{2} \cdot \exp \left(j k_{z} z_{1}\right)-P_{1} * H_{1} \cdot \exp \left(j k_{z} z_{2}\right)}{P_{1} * H_{1} \cdot \exp \left(-j k_{z} z_{2}\right)-P_{2} * H_{2} \cdot \exp \left(-j k_{z} z_{1}\right)}
$$

Where, $P_{1,2}$ and $P_{1,2}^{\prime}$ represent $P_{m}\left(k_{x}, k_{y}, z_{1,2}\right)$ and $P_{m}^{\prime}\left(k_{x}, k_{y}, z_{1,2}\right), H_{1,2}$ represents $H_{m}\left(k_{x}, k_{y}, z_{1,2}\right)$.

Comparing Eq. 1 and Eq. 6, it is sure that there is a difference between the sound reflection coefficient reconstructed by NAH in the free-field and in a finite field with multi-path interferences.

\section{Holography measurement data pre-processing method}

In a rectangular parallelepiped tank which boundaries are not completely sound absorption, for example, parts of the multi-path interferences are shown in Fig. 1.

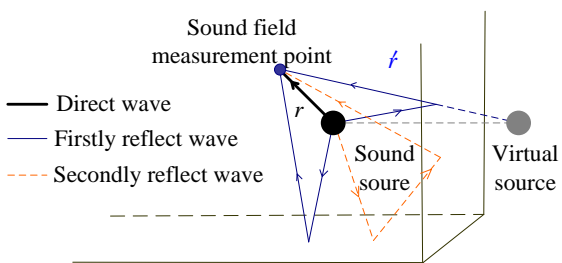

Figure 1 the diagram of multi-path interferences in a finite space

In many cases, the channel impulse response functions of the measurement environments are difficult to be estimated. It is assumed that the holography sound pressures in the finite space have been measured whether the test material is placed or not. The complex sound pressures on the measurement holography planes can be expressed as follows:

$$
\begin{aligned}
& p_{0}^{\prime}(\omega ; x, y, z)=p_{0}(\omega ; x, y, z) \cdot h_{0}(\omega ; x, y, z) \\
& p_{m}^{\prime}(\omega ; x, y, z)=p_{m}(\omega ; x, y, z) \cdot h_{m}(\omega ; x, y, z)
\end{aligned}
$$


Where, $p_{0}^{\prime}(\omega ; x, y, z)$ is the complex sound pressure when there is no material in finite space, $p_{0}(\omega ; x, y, z)$ is the complex sound pressure when there is no material in free space, $h_{0}(\omega ; x, y, z)$ is the channel impulse response functions when there is no material. $p_{m}^{\prime}(\omega ; x, y, z), p_{m}(\omega ; x, y, z)$, and $h_{m}(\omega ; x, y, z)$ are the physical parameters when the material is placed in the measurement environment.

It is approximately assumed that the direct wave signal is only relative to the measurement position. If the steady-state sound source signal is marked as $s(t)$, the estimation value of direct wave in accordance with spherical wave attenuation law can be expressed as follows $[4,5]$ :

$$
\hat{p}_{0}(t ; x, y, z)=\frac{1}{r} \cdot s(t-r / c)
$$

If the complex sound pressure format of $\hat{p}_{0}(t ; x, y, z)$ is marked as $\hat{p}_{0}(\omega ; x, y, z)$, the estimation value of $h_{0}(\omega ; x, y, z)$ can be obtained by Eq. 7:

$$
\hat{h}_{0}(\omega ; x, y, z)=p_{0}^{\prime}(\omega ; x, y, z) / \hat{p}_{0}(\omega ; x, y, z)
$$

If the dimension of the material is much smaller than the measurement field, it can be regarded that there is little effect on the channel impulse response function when the material is placed in the field:

$$
h_{0}(\omega ; x, y, z) \cong h_{m}(\omega ; x, y, z)
$$

Therefore, $\hat{p}_{m}(\omega ; x, y, z)$ can be calculated according to Eq. 7 and Eq. 8 [3]:

$$
\begin{aligned}
\hat{p}_{m}(\omega ; x, y, z) & =p_{m}^{\prime}(\omega ; x, y, z) / \hat{h}_{m}(\omega ; x, y, z)=p_{m}^{\prime}(\omega ; x, y, z) / \hat{h}_{0}(\omega ; x, y, z) \\
& =p_{m}^{\prime}(\omega ; x, y, z) \cdot p_{0}^{\prime}(\omega ; x, y, z) / \hat{p}_{0}(\omega ; x, y, z)
\end{aligned}
$$

Thus, if the measurement is taken in finite space, the holography measurement results should be pre-processed by Eq.12. Then, the pre-processed results are decomposed into plane wave components. Finally, the material sound reflection coefficient is reconstructed by Eq. 1 and Eq. 2 .

\section{The holography measurement experiment in an anechoic tank}

In order to illustrate the effectiveness of the data pre-processing method which is proposed in this paper, the experiment was taken in an anechoic tank. The tank is approximately a cylindrical structure, its inner diameter is about $4 \mathrm{~m}$, and the inner length is about $20 \mathrm{~m}$. A standard steel plane is chosen as the material to be test, and its size is $1.5 \mathrm{~m} \times 1.2 \mathrm{~m} \times 6 \mathrm{~mm}$. The experiment is carried out at atmospheric pressure. The experiment measurement system is shown as Fig. 2.

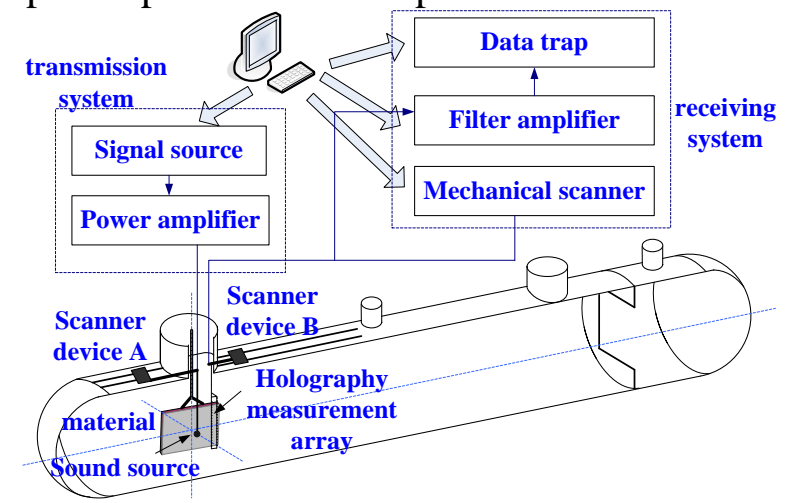

Figure 2 the diagram of measurement system

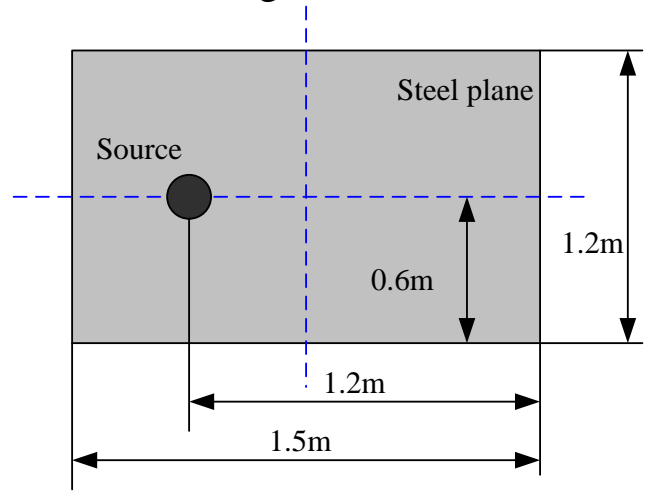

Figure 3 the location of sound source

The sound source is a spherical transducer. In order to obtain reflection coefficients at more angles, the sound source is not placed facing the center of the sound material. The specific location is shown as Fig. 3. 
The sound-absorbing materials are laid on the inner wall of the tank but not on the positions such as the mechanical guides and openings. Therefore, the multi-path interferences inside the tank are difficult to estimate. Use the sound source to emit white noise signals, then the reverberation time of the tank is measurement by the PULSE dynamic analyzer. The reverberation time of $1 / 3$ octave bandwidth is shown as Fig. 4.

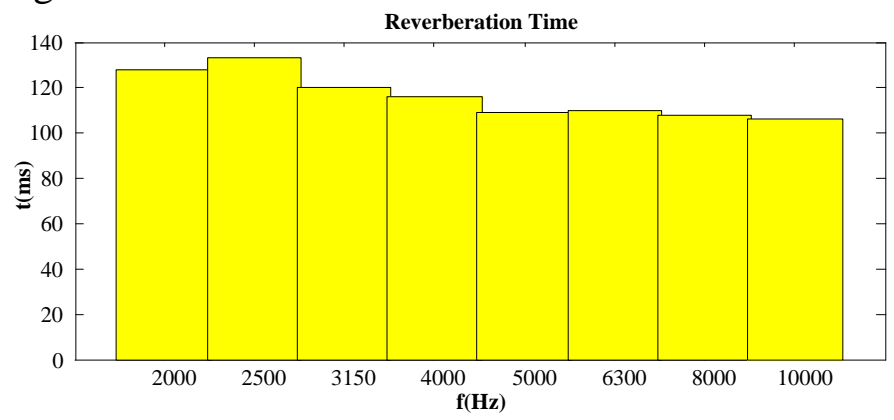

Figure 4 the reverberation time of the anechoic tank

The reverberation radius $r_{h}$ of the anechoic tank is calculated according to the following formula:

$r_{h}=0.05 \sqrt{\frac{V}{\pi T_{60}}} \quad\left(V \approx \pi * 2^{2} * 20=251.3 m^{3}\right)$

The calculated results of reverberation times and reverberation radius are shown in Table 1:

Table 1 Calculated results of reverberation times and reverberation radius

\begin{tabular}{c|c|c|c|c|c|c|c|c}
\hline frequency $f(\mathrm{~Hz})$ & 2000 & 2500 & 3150 & 4000 & 5000 & 6300 & 8000 & 10000 \\
\hline Reverberation time $T_{60}(\mathrm{~ms})$ & 128 & 133 & 120 & 116 & 109 & 110 & 108 & 106 \\
\hline Reverberation radius $r_{h}(\mathrm{~m})$ & 1.250 & 1.226 & 1.291 & 1.313 & 1.355 & 1.348 & 1.361 & 1.374 \\
\hline
\end{tabular}

In the anechoic tank: when $r=r_{h}$, the direct sound is equal to the reverberation sound. When $r>r_{h}$, the measurement domain is in the reverberation control area, the reverberation sound is more important. When $r<r_{h}$, the direct sound is more important [6].

As can be seen from Table 1, the reverberation time in each frequency band is about $110 \mathrm{~ms}$, the reverberation radius is about $1.3 \mathrm{~m}$. In conjunction with Fig. 3, parts of the distances between measurement points and the source are larger than reverberation radius. Therefore, the multi-path interferences can not be ignored, the holography data are needed to be measured at the same positions whether the material is placed in the tank or not.

Use the sound source to transmit sine signal. The complex sound pressure measurement array is moved to receive holography data. The moving step is $0.03 \mathrm{~m}$, the holography planes are rectangle $(1020 \mathrm{~mm} \times 1500 \mathrm{~mm})$. The distances between the two planes and the material to be test are respectively $3.5 \mathrm{~cm}$ and $7.5 \mathrm{~cm}$. The spacing of sound source and the material is $15 \mathrm{~cm}$ [1]. The measurement data are pre-processed by Eq. 12. The pre-processed data are filtered in wave number domain and zeros processed. Then the reflection coefficient of the material is reconstructed by NAH technology. The reconstructed results and the errors of the reconstructed results are shown as Fig. 5 and Fig. 6.

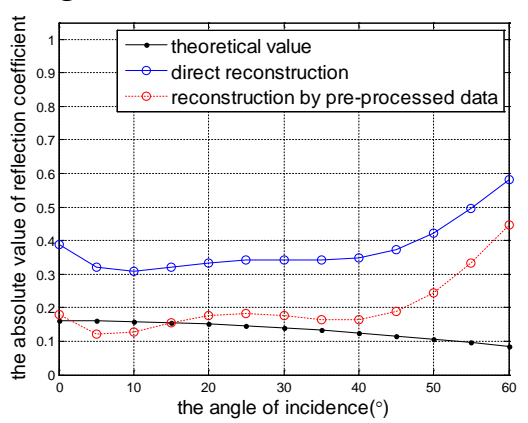

(a) $\mathrm{f}=2000 \mathrm{~Hz}$

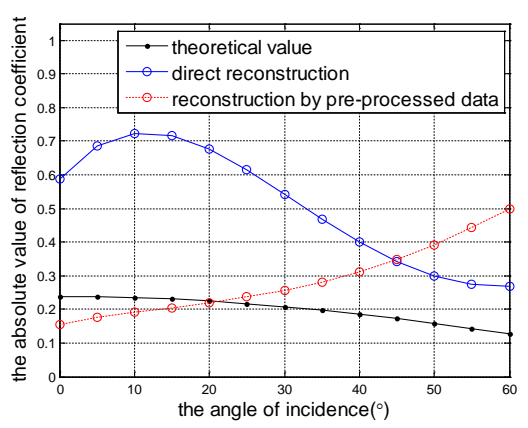

(b) $\mathrm{f}=3000 \mathrm{~Hz}$

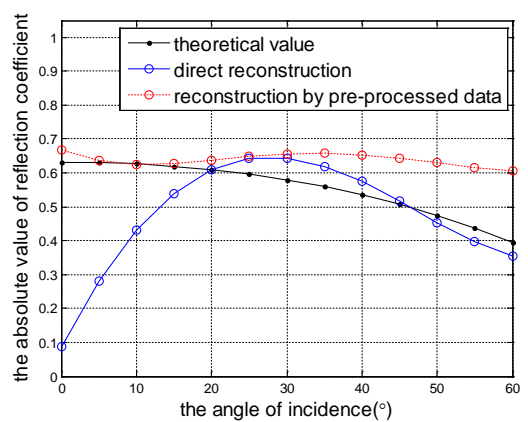

(c) $\mathrm{f}=10000 \mathrm{~Hz}$

Figure 5 the comparative analysis of the measurement results with the theoretical value 


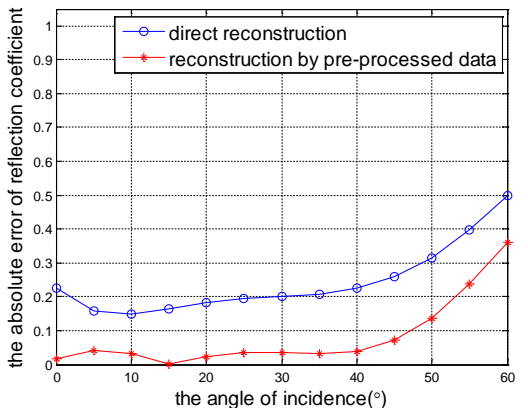

(a) $\mathrm{f}=2000 \mathrm{~Hz}$

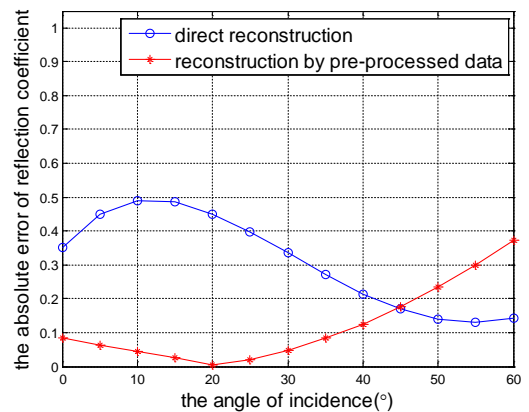

(b) $\mathrm{f}=3000 \mathrm{~Hz}$

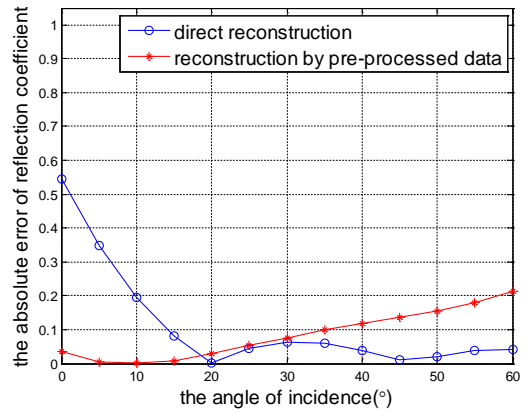

(c) $\mathrm{f}=10000 \mathrm{~Hz}$

Figure 6 the absolute error of reconstructed results

The mean square errors of the reflection coefficient reconstructed results $\left(0^{\circ} \sim 60^{\circ}\right)$ are shown in Table 2.

Table 2 the mean square errors of the reflection coefficient reconstructed results

\begin{tabular}{c|c|c|c}
\hline $\mathrm{f}(\mathrm{Hz})$ & 2000 & 3000 & 10000 \\
\hline Directly reconstruction & 0.2638 & 0.3372 & 0.1916 \\
\hline Reconstruction by pre-processed data & 0.1297 & 0.1645 & 0.1093 \\
\hline
\end{tabular}

As can be seen from the Fig. 5, Fig. 6 and Table 2, the data pre-processing method proposed in this paper can greatly improve the reconstruction accuracy of the acoustic reflection coefficient.

\section{Conclusions}

In this paper, in order to solve the multi-path interferences problem in finite space, a pre-processing method for holography data is proposed. As can be seen from the experimental data processing results in an anechoic tank, the pre-processing method is effective to suppress the multi-path interferences in finite space, and the reconstruction accuracy of NAH method is greatly improved. Because the requirements for the measurement environment are not very strict, this pre-processing method will have broad application prospects.

\section{Acknowledgments}

This work was supported by the National Natural Science Foundation of China (11404079) and Natural Science Foundation of Heilongjiang Province of China (LC2013C03).

\section{References}

[1] Gao Y, Wang Y, Liu Y W, Shang D J. Experimental investigation on reflection coefficients reconstruction by NAH in semi-space[J]. Technical Acoustics., 2007, 26(5): 990-991.

[2] He Y A, Li R, He Z Y. A technique of reconstructing reflection coefficients at arbitrary angles of incidence[J]. ACTA ACOUSTICA. , 1996,21(6): 928-934

[3] Xiao Y, Shang D J, Hu H H, Liu Y W. Investigation on reconstructing sound reflection coefficient of materials by NAH in finite space[J]. Technical Acoustics., 2013, 32(5): 164-166.

[4] Blaine M. Harker, Brian E. Anderson. Optimization of the array mirror for time reversal techniques used in a half-space environment[J]. J. Acoust. Soc. Am., 2013, 133 (5): 351-357.

[5] Shi J, Yang D S, Liu B S. Radiated noise sources near-field location based on virtual time reversal mirror[J]. ACTA ARMAMENTARII., 2008, 29(10): 1215-1219.

[6] Shang D J, Li Q, Shang D J, Hou B L. Measurement of the radiated power of sound sources in a reverberation pool[J]. Journal of Harbin Engineering University., 2010,37(7): 938-944. 